REGARDS

SUR L'ECONOMIE ALLEMAND

BULLETIN ECONOMIQUE DU CIRAC
Regards sur l'économie allemande

Bulletin économique du CIRAC

116-117| 2015

Varia

\title{
Anticiper le changement
}

Isabelle Bourgeois

\section{CpenEdition}

Journals

Édition électronique

URL : https://journals.openedition.org/rea/4809

DOI : $10.4000 /$ rea. 4809

ISSN : 1965-0787

Éditeur

CIRAC

\section{Édition imprimée}

Date de publication : 1 juillet 2015

Pagination : 3-4

ISSN : 1156-8992

\section{Référence électronique}

Isabelle Bourgeois, «Anticiper le changement », Regards sur l'économie allemande [En ligne], 116-117 | juillet 2015, mis en ligne le 01 juillet 2015, consulté le 28 juin 2022. URL : http:// journals.openedition.org/rea/4809; DOI : https://doi.org/10.4000/rea.4809 


\section{Anticiper le changement}

Depuis le début du printemps, les révisions à la hausse s'enchaînent. Alors que le Conseil des Sages prévoyait encore, en novembre 2014, une croissance de 1,0\% pour 2015, il a doublé ses prévisions en mars 2015 et s'attend désormais à $1,8 \%$. II a été suivi en avril par le gouvernement fédéral qui voit le PIB augmenter de 1,8\% (et non plus de 1,5\%). Quant au rapport de printemps des Instituts, présenté le 16 avril, il table d'entrée sur une croissance de 2,1\% pour l'année en cours. Seule la Bundesbank reste, comme toujours, prudente : dans son rapport de juin, elle prévoit désormais une hausse du PIB de 1,7\% (1,5\% en données CVS).

L'économie allemande est sortie plus rapidement que prévu du creux conjoncturel du milieu de 2014 : alors que l'année avait bien commencé $(+0,8 \%)$, l'activité s'était ralentie aux $2^{\mathrm{e}}$ et $3^{\mathrm{e}}$ trimestres $(-0,1 \%$ puis $+0,1 \%)$, avant de reprendre au dernier (+0,7\% ; Destatis). Au $1^{\text {er }}$ trimestre 2015 , la croissance n'a certes été que de $0,3 \%$ par rapport au dernier de 2014, mais de 1,1\% en comparaison annuelle. L'économie allemande a retrouvé un bon rythme de croisière grâce à une consommation intérieure dynamique et un environnement favorable. Le frémissement au sein de la zone Euro et, surtout, la dévalorisation de l'Euro face au dollar portent le commerce extérieur ; au plan domestique, l'excellente tenue du marché de l'emploi comme le faible prix du pétrole qui réduit l'inflation tirent la demande intérieure.

Les Allemands sont entrés dans l'année 2015 d'un bon pied, comme le révélait le sondage effectué tous les ans en décembre depuis 1949 par l'Institut für Demoskopie d'Allensbach (IfD) auprès d'un échantillon représentatif de la population : $56 \%$ des personnes interrogées abordaient 2015 " pleines d'espoir », confiantes dans la stabilité de leur emploi et le dynamisme économique de leur pays (IfD, 29-12-2014). Ce taux de confiance a rarement été aussi élevé depuis les années 1990, où il n'a atteint un niveau similaire qu'au moment de l'Unité et de la « bulle Internet ». Les indicateurs du climat des affaires, dont l'indice ifo, abondent dans le même sens : les entreprises se montrent elles aussi résolument optimistes quant à l'évolution de l'activité.

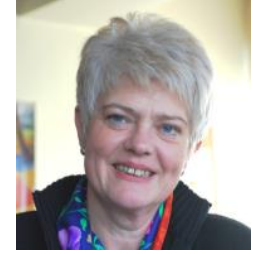

Isabelle Bourgeois, Rédactrice en chef

2015 a bien démarré

Confiance des acteurs économiques

\begin{tabular}{|c|c|c|c|c|}
\hline \multicolumn{5}{|c|}{ Prévisions actualisées du Conseil des Sages } \\
\hline & 2013 & 2014 & $\begin{array}{c}2015 \mathrm{P} \\
\text { mars } 2015\end{array}$ & $\begin{array}{c}2015 P \\
\text { nov. } 2014\end{array}$ \\
\hline \multicolumn{5}{|l|}{ évolution } \\
\hline PIB (\%) & 0,1 & 1,6 & 1,8 & 1,0 \\
\hline Consommation (\%) & 0,8 & 1,2 & 2,1 & 1,4 \\
\hline privée (\%) & 0,8 & 1,2 & 2,4 & 1,5 \\
\hline publique (\%) & 0,7 & 1,1 & 1,2 & 1,2 \\
\hline \multicolumn{5}{|l|}{ Investissements } \\
\hline biens d'équipement (\%) & $-2,4$ & 4,3 & 3,8 & 3,9 \\
\hline construction (\%) & $-0,1$ & 3,6 & 1,8 & 2,0 \\
\hline Demande intérieure (\%) & 0,7 & 1,3 & 1,8 & 1,5 \\
\hline Part de l'excédent commercial dans le PIB (points de pourcentage) & $-0,5$ & 0,4 & 0,1 & $-0,4$ \\
\hline exportations (\%) & 1,6 & 3,9 & 5,1 & 3,6 \\
\hline importations (\%) & 3,1 & 3,4 & 5,5 & 5,1 \\
\hline Prix à la consommation (\%) & 2,0 & 1,5 & 1,0 & 1,3 \\
\hline \multicolumn{5}{|l|}{ état } \\
\hline Actifs occupés (milliers) & 42281 & 42652 & 42956 & 42795 \\
\hline Actifs occupant un emploi soumis à cotisations sociales (milliers) & 29713 & 30207 & 30594 & 30474 \\
\hline Chômeurs indemnisés (milliers) & 2950 & 2898 & 2822 & 2931 \\
\hline Taux de chômage (Agence fédérale pour l'emploi) (\%) & 6,9 & 6,7 & 6,5 & 6,7 \\
\hline Prix à la consommation & 1,5 & 0,9 & 0,3 & 1,3 \\
\hline Déficit public (\% du PIB) & 0,1 & 0,6 & 0,4 & $-0,0$ \\
\hline
\end{tabular}

Source : Sachverständigenrat zur Begutachtung der gesamtwirtschaftlichen Entwicklung, Aktualisierung der Konjunkturprognose, 26 mars 2015.

Malgré un léger recul au mois de mars (-0,5\%), la production industrielle, tirée par les biens d'investissement surtout, reste bien orientée dans l'ensemble en avril (+0,6\%; Destatis). Mais, souligne la Bundesbank, elle ne se situe depuis quelque temps qu'au niveau qu'elle atteignait en 2007 , soit avant la crise de la finance mondiale, et le taux d'utilisation des capacités ne laisse guère présager pour l'instant un effort accru d'extension; c'est là une des raisons qui expliquent la relative faiblesse des investissements. Quoi qu'il en soit, les commandes affluent, notamment en provenance de
Production industrielle bien orientée 
La croissance portée par la consommation

Le « doux poison" d'un Euro dévalué l'étranger, et surtout de la zone Euro (+8\%; Bundesbank), où a été enregistrée une forte demande dans le secteur de l'aéronautique. Sur le marché domestique, les commandes se stabilisent à $0,5 \%$ après un pic exceptionnel dans le secteur automobile le mois précédent. Dans le domaine des biens d'investissement $(+2,75 \%)$, ce sont la construction mécanique et l'automobile qui dominent. Mais les plus dynamiques sont les biens de consommation (+4\%). Quant au BTP, où l'activité s'était ralentie en mars après un hiver clément, il a connu une nette progression des commandes au $1^{\text {er }}$ trimestre $(+8,5 \%$; Bundesbank) sous l'effet d'une forte demande publique notamment. Si les exportations continuent de progresser en avril (+1,9\%), les importations ont reculé $(-1,3 \%$; ministère fédéral de l'Economie) principalement sous l'impact de la dévaluation de l'Euro. Au total, l'excédent commercial, bien que toujours élevé (217 milliards $€$ en 2014), ne contribue guère actuellement à la croissance.

Celle-ci est portée par la consommation privée : au $1^{\text {er }}$ trimestre, elle a crû de 0,6\% par rapport au précédent (mais de 2,4\% en comparaison annuelle ; Destatis). Cette tendance s'était accélérée au deuxième semestre 2014 avec la baisse du prix du pétrole qui avait accru le revenu disponible des ménages, tout autant que la hausse des transferts sociaux et la faible inflation. Plus fondamentalement, l'optimisme des ménages est entretenu par la compétitivité de l'économie allemande, la politique de consolidation budgétaire et la robustesse du marché de l'emploi : le nombre d'actifs occupés devrait friser cette année les 43 millions, et le taux de chômage, avec moins de $5 \%$ (critères OIT), se maintenir à un niveau historiquement bas.

Si les fondamentaux de l'économie allemande sont bons, les diverses prévisions de croissance n'en soulignent pas moins l'existence d'un certain nombre de risques externes planant sur cette économie ouverte : flambée du prix du pétrole, « insolvabilité de la Grèce " (Conseil des Sages), "vulnérabilité de certaines économies émergentes », “tensions géopolitiques », sans parler d'une "forte réévaluation de l'Euro » (Bundesbank; communiqué du 05-06-2015). Car la croissance allemande est aussi dopée actuellement par un environnement exceptionnellement favorable. Ce « doux poison » (Handelsblatt, 20-01-2015) que sont les faibles prix pétroliers et la politique monétaire accommodante de la BCE incite à se reposer sur ses lauriers. Les entreprises industrielles, exposées à la concurrence mondiale, sauront assurément redoubler d'efforts en matière d'innovation pour rester compétitives, d'autant qu'elles ne manquent pas de capitaux propres et que l'accès au crédit bancaire est aisé.

MAIS INVESTIRONT-ELLES PLUS POUR AUTANT ? Le rapport de printemps des instituts économiques, qui se penche sur la question, rappelle que, malgré un léger mieux d'ici la fin de l'année, les investissements privés n'atteindront qu'à peine le niveau de l'année 2008 en Allemagne, soit le pic d'avant la crise. Ce rattrapage différé est un phénomène constaté dans de nombreuses économies européennes ; il se conjugue avec les incertitudes liées aux économies émergentes, au conflit russo-ukrainien et à l'avenir de la zone Euro. Plus spécifique à l'économie allemande est le fait que les conditions-cadre que réserve à l'activité la Grande coalition ne sont guère incitatives pour l'investissement à l'intérieur du pays. Sa politique est en effet "contradictoire », mêlant à quelques mesures favorables à la croissance des mesures de nature à l'inhiber, comme le SMIC légal qui assèche le vivier de main-d'œuvre «dont la productivité est inférieure à ce seuil ", la retraite à 63 ans qui prive les entreprises de compétences clés, l'encadrement des loyers qui rend imprévisible le marché immobilier, ou encore les incessants réajustements réglementaires de la transition énergétique.

"Le site Allemagne doit devenir plus attractif pour les investisseurs », insistent les Instituts, soulignant que le gouvernement fédéral a présenté un train de mesures en faveur de l'investissement, centrées principalement sur la recherche et l'innovation, les infrastructures et sur des gains d'efficience dans l'administration. Or, rappellent-ils, en matière d'investissements publics, ce n'est "pas la quantité qui compte, mais la qualité ». Et surtout, " bien plus stratégiques encore que les investissements dans le béton sont ceux dans les têtes "; il ne sert à rien d'augmenter les dépenses dans l'éducation et la recherche, seule une approche qualitative, c'est-à-dire en termes de calcul coûts/bénéfices, générera la hausse indispensable des qualifications. La seule politique d'attractivité adaptée est la poursuite des réformes structurelles, notamment fiscales, rappellent tous les experts. Et bien sûr la réduction de la dette publique - $a$ fortiori pour une économie confrontée au vieillissement démographique.

(I. B., 23/06/2015) 